\title{
Tilling and Eco-Tilling - A Reverse Genetic Approach for Crop Improvement
}

\author{
Azra Khan ${ }^{1 *}$, Ishfaq Abidi ${ }^{3 *}$, M.A. Bhat ${ }^{1}$, Z.A. Dar ${ }^{2}$, Gowhar Ali ${ }^{3}$, \\ Asif B. Shikari ${ }^{4}$ and M.A. Khan ${ }^{1}$
}

${ }^{1}$ Division of Genetics and Plant Breeding, Faculty of Agriculture Wadura, Sher-i-Kashmir

University of Agricultural Sciences and Technology of Kashmir, India

${ }^{2}$ Division of Genetics and Plant Breeding, Dryland Agriculture Research Station, Budgam, Sher-i-Kashmir University of Agricultural Sciences and Technology of Kashmir, India

${ }^{3}$ Division of Genetics and Plant Breeding, Sher-i-Kashmir University of Agricultural Sciences and Technology of Kashmir, Shalimar, India

${ }^{4}$ MCRS Sagam, Sher-i-Kashmir University of Agricultural Sciences and Technology of Kashmir, India

*Corresponding author

\begin{tabular}{|l|}
\hline K e y w o r d s \\
TILLING, ECO- \\
TILLING, Mutagenesis, \\
DNA polymorphism, \\
Induced
\end{tabular}

\section{Introduction}

A new era of genomics for plant science
research has opened due the complete genome
sequencing projects of Arabidopsis thaliana

A new era of genomics for plant science
research has opened due the complete genome
sequencing projects of Arabidopsis thaliana

A new era of genomics for plant science
research has opened due the complete genome
sequencing projects of Arabidopsis thaliana
Targeting Induced Local Lesions in Genome (TILLING) is a general reverse technique that combines chemical mutagenesis with PCR based screening to identity point mutations in regions of interest. The strategy works with a mismatch-specific endonuclease to detect induced or natural DNA polymorphisms in gene of interest. The main principles of TILLING include making EMS-mutagenized population, targeting gene of interest with PCR primers and searching rare mutants among many individuals. A study based on applying TILLING to elucidate gene function in a chemically induced sorghum mutant population showed that total of five mutants were detected for four gene targets. High throughput TILLING was demonstrated applicable to maize, with a large genome but with limited genetic resources currently available. This study also concluded public TILLING service for maize, modelled on the existing Arabidopsis TILLING Project. The basic applications of ECO-TILLING include mapping, association analysis, mutation profiling and biodiversity. The main advantage of TILLING is its ability in determining the spectrum of variation and for genetic mapping based on linkage association analysis. The technique is a however cost effective too. One of the basic disadvantages of TILLING is the low rate of induction of mutation and requirement of skilful labours. and rice. The sequence information available in public database has highlighted the need to develop genome scale reverse genetic strategies for functional analysis (Till et al., 2003). As most of the phenotypes are obscure, 
the forward genetics can hardly meet the demand of a high throughput and large-scale survey of gene functions. Targeting Induced Local Lesions in Genome TILLING is a general reverse genetic technique that combines chemical mutagenesis with PCR based screening to identity point mutations in regions of interest (McCallum et al., 2000). This strategy works with a mismatch-specific endonuclease to detect induced or natural DNA polymorphisms in genes of interest. A newly developed general reverse genetic strategy helps to locate an allelic series of induced point mutations in genes of interest. It allows the rapid and inexpensive detection of induced point mutations in populations of physically or chemically mutagenized individuals.

To create an induced population with the use of physical/chemical mutagens is the first prerequisite for TILLING approach. Most of the plant species are compatible with this technique due to their self-fertilized nature and the seeds produced by these plants can be stored for long periods of time (Borevitz et al., 2003). The seeds are treated with mutagens and raised to harvest M1 plants, which are consequently, self-fertilized to raise the M2 population. DNA extracted from M2 plants is used in mutational screening (Colbert et al., 2001). To avoid mixing of the same mutation only one M2 plant from each M1 is used for DNA extraction (Till et al., 2007). The M3 seeds produce by selfing the M2 progeny can be well preserved for long term storage. Ethyl methane sulfonate (EMS) has been extensively used as a chemical mutagen in TILLING studies in plants to generate mutant populations, although other mutagens can be effective. EMS produces transitional mutations $(\mathrm{G} / \mathrm{C}, \mathrm{A} / \mathrm{T})$ by alkylating $G$ residues which pairs with $T$ instead of the conservative base pairing with C (Nagy et al., 2003). It is a constructive approach for users to attempt a range of chemical mutagens to assess the lethality and sterility on germinal tissue before creating large mutant populations.

\section{History and overview}

TILLING first began in the late 1990's by a graduate student, Claire McCallum (and collaborators from Fred Hutchinson Cancer Research Center and Howard Hughes Medical Institute), who worked on characterizing the function of two chromomethylase genes in Arabidopsis (Henicoff et al., 2004).Claire McCallum utilized reverse genetic approaches such as T-DNA lines and antisense RNA, but was unable to successfully apply these approaches to characterize CMT2. The approach that was successful turned out to be what is now known as TILLING (Targeting Induced Local Lesions in Genomes). This was accomplished by pooling chemically induced mutagenized plants together, amplifying the region of interest, creating heteroduplexes among the pooled DNA, and performing dHPLC (denaturing high performance liquid chromatography) to detect the mutants by chromatographic alterations(McCallum et al., 2000).

Since the inception of this method, TILLING has been streamlined, automated, and utilized in many plant and animal taxa. TILLING, which is a reverse genetic high throughput approach, aims to identify SNPs (single nucleotide polymorphisms) and / or INDELS (insertions/ deletions) in a gene/genes of interest from a mutagenized population. Therefore, the first step in TILLING is the creation of a mutagenized population, which is often accomplished by treatment with a chemical mutagen such as EMS. Many plant species are well suited for this strategy because they can be self-fertilized and seeds can be stored for long periods of time (Henicoff et al., 2004; McCallum et al., 2000). However, multiple strategies also exist 
for the creation of mutant populations in animal species (Sood et al., 2006; Gilchrist et al., 2006). In plants, seeds are treated with EMS and grown out to produce M1 plants, which are subsequently self-fertilized to produce the M2 generation. Leaf tissues from M2 plants are collected for DNA extraction and then used for mutational screening (Colbert et al., 2001). To avoid sampling of the same mutation only one M2 individual from each M1 is chosen for DNA extraction (Till et al., 2007). The M2 progeny can be self-fertilized and the resulting M3 seed can be preserved in long term storage (Till et al., 2003). EMS has been widely used as a chemical mutagen in TILLING in both plant and animal studies to generate mutant populations, although other chemical mutagens can be effective. EMS typically produces transition mutations (G/C: A/T) because it alkylates $\mathrm{G}$ residues (Comia et al., 2006) and the alkylated $G$ residue pairs with $\mathrm{T}$ instead of the conventional base pairing with C (Henicoff et al., 2003).

The various steps for TILLING process are mentioned by Lightner et al., 1998 for Arabidopsis as:

\section{EMS mutagenesis}

DNA preparation and pooling of individuals

PCR amplification of a region of interest with fluorescently tagged primers

Denaturation and annealing to allow formation of heteroduplexes at the site of mutation

Resultant double-stranded products are digested with CEL I, which cleaves on of the two strands at the heteroduplex mismatches

Cleaved products are detected on polyacrylamide denaturing gels.

\section{Case study 1}

A study based on applying TILLING to elucidate gene function in a chemically induced sorghum mutant population showed that total of five mutants were detected for four gene targets (Zhanguo et al., 2008). A total of 768 mutant lines were assayed for mutation induction in the target genes. Four gene targets were selected based on their potential contribution to bioenergy, nutrition, and agronomic performance for high throughput TILLING. Eight-fold pools of genomic DNA from leaf tissues of M2 plants were used for TILLING. Mutagenesissorghum inbred line $\mathrm{BT}^{*} 623$ was used to generate the mutant populations using EMS ( 0.1 to $0.6 \%$ mutagenesis).

A sorghum mutant population consisting of 1,600 lines was generated from the inbred line BTx623 by treatment with the chemical agent ethyl methanesulfonate (EMS). Numerous phenotypes with altered morphological and agronomic traits were observed from M2 and M3 lines in the field. A subset of 768 mutant lines was analyzed by TILLING using four target genes. A total of five mutations were identified resulting in a calculated mutation density of $1 / 526 \mathrm{~kb}$. Two of the mutations identified by TILLING and verified by sequencing were detected in the gene encoding caffeic acid $O$-methyltransferase $(C O M T)$ in two independent mutant lines. The two mutant lines segregated for the expected brown midrib (bmr) phenotype, a trait associated with altered lignin content and increased digestibility. Moreover 0.25\% EMS concentration is most effective.

\section{Case study 2}

High throughput TILLING was demonstrated applicable to maize, with a large genome but with limited genetic resources currently available (Bradley J Till et al., 2004). 
Demonstrated that high-throughput TILLING is applicable to maize, an important crop plant with a large genome but with limited reversegenetic resources currently available. They screened pools of DNA samples for mutations in 1-kb segments from 11 different genes, obtaining 17 independent induced mutations from a population of 750 pollen-mutagenized maize plants. One of the genes targeted was the DMT102 chromomethylase gene, for which they obtained an allelic series of three missense mutations that are predicted to be strongly deleterious.

\section{Applications}

Major areas of applications are

\section{Functional genomics}

The identification of numerous mutations in target region of genome. Construction of TILLING library is useful for scientists to search for mutations in gene of interest. TILLING offers a way to investigate target GOI in any crop of interest without first having knowledge of gene product.

\section{Genetic engineering}

Agricultural interest in producing phenotypic variants without introducing foreign DNA of any type into plants genome. T-DNA/ Transposon insertions are used to obtain specific gene knockouts but practically limited to some crops only. TILLING is in front of transgene, as consists of identification of numerous mutations within a targeted region of whole genome.

\section{Evaluation of genetic diversity of natural populations}

Alternative to wild relatives, TILLING is used to introduce useful genetic variation of elite germplasm. Also applicable in a population which has several pre-existing polymorphism for developing SNPs.

\section{ECO-TILLING}

ECO-TILLING (Ecotype Targeting Induced Local Lesions IN Genomes), a rapid, inexpensive and well-established allele mining approach is found much proficient in large-scale mining and high-throughput genotyping of novel natural and functional allelic variants (without prior knowledge of SNP alleles) of known and candidate genes related to useful agronomic traits in diverse crop germplasm accessions (McCallum et al., 2000; Comai et al., 2004; Till et al., 2006, 2007, 2010; Raghavan et al., 2007; Wang et al., 2010; Xia et al., 2012). The first publication of the ECO-TILLING method in which TILLING was modified to mine for natural polymorphisms was in 2004 for Arabidopsis. ECO-TILLING is similar to TILLING, except that its objective is to identify natural genetic variation as opposed to induced mutations. Many species are not amenable to chemical mutagenesis, therefore ECO-TILLING can aid in the discovery of natural variants. ECO-TILLING is a means to determine the extent of natural sequence variation across many germplasms, enabling both SNP discovery and haplotyping (Comia et al., 2004). This technique is now applied to rice, maize, lotus, poplar (Gilchrist et al., 2006), Brassica, zebrafish, Drosophila, Caenorhabditis and human (Till et al., 2006), indicating its broad applicability. The implication of ECO-TILLING to identify potential novel functional alleles in the known and candidate genes/transcription factors (TFs) regulating qualitative and quantitative agronomic traits by association/genetic mapping is well-documented for expediting the genetic enhancement of crop plants (Mejlhede et al., 2006; Barkley and Wang, 2008; Ibiza et al., 2010; Negrao et al., 2011; $\mathrm{Yu}$ et al., 2012; Frerichmann et al., 2013). 
ECO-TILLING usually employs a mismatchspecific CEL-I nuclease to cleave the PCR amplified fragments at the site of heteroduplex formation involving nucleotide (SNP-allelic) polymorphism. Most of the ECO-TILLING studies utilize the advanced genotyping platforms (LICOR NEN Model 4300 DNA Analyzer, Transgenomic WAVEHS denaturing high performance liquid chromatography, ABI 377 sequencer and eGene capillary electrophoresis systems) for efficient resolution of fluorescent dye (IRDye 700/800 and SYBR green)-labeled CEL-I cleaved heteroduplex PCR amplified fragments. Consequently, these efforts led to the discovery and genotyping of novel potential alleles specifically derived from the trait-associated known and candidate genes in natural population of diverse crop plants (Perry et al., 2003; Caldwell et al., 2004; Comai et al., 2004; Henikoff et al., 2004; Yang et al., 2004; Suzuki et al., 2005). The added-advantage of agarose gel-based ECOTILLING vis-à-vis the commonly utilized LICOR genotyper for large-scale mining and genotyping of allelic variants in accessions exhibiting low level polymorphism, is welldemonstrated in many crop plants (Raghavan et al., 2007; Negrao et al., 2011; Yu et al., 2012). This is merely because efficacy of an agarose gel-based ECO-TILLING approach in precise resolution of unlabeled CEL Icleaved heteroduplex PCR amplified fragments by a simpler, economical and timesaving agarose gel-based detection assay as compared to a standard ECO-TILLING method that requires labeled CEL I-cleaved heteroduplex PCR amplicons for resolution in a LICOR genotyper. The broader utility and deployment of this agarose gel-based ECOTILLING approach in manifold large-scale genotyping applications is well-documented by the research laboratories with minimal resources (Raghavan et al., 2007; Negrao et al., 2011; Yu et al., 2012). This includes understanding the natural allelic diversity, population genetic structure and domestication pattern among accessions, molecular mapping and genetic association analysis for identification of potential molecular tags like alleles and genes/QTLs (quantitative trait loci) governing vital agronomic traits and marker-assisted breeding for selecting desirable accessions for crop genetic improvement.

These methods are very useful in rapid and cheap detection of small mutations or natural polymorphisms. Mutations are the primary factors in nature to bring about variations. TILLING method can be used to generate disease resistant plants. This can be applied for Diploid or Allohexaploid and may provide a rapid approach to reverse genetics by the identification of induced and naturally occurring variation in many plant species. Cost effective method than Genetic engineering and has no associated bio-safety issues. But the problem lies in the fact that the rate of induction of mutation is low, requires skilful labours and moreover a mutagenized organism must be kept alive long enough to screen mutant population in vegetatively propagated plant species.

\section{References}

Barkley N.A and Wang M.L. 2008. Application of TILLING and Eco TILLING as Reverse Genetic Approaches to Elucidate the Function of Genes in Plants and Animals. Current Genomics.

Borevitz, J. O., Liang, D., Plouffe, D., Chang, H.S., Zhu, T., Weigel, D., Berry, C.C., Winzeler, E. and Chory, J. 2003. Largescale identification of single-feature polymorphisms in complex genomes. Genome Research 13(3): 513-523.

Bradley, J. T., Reynolds, S.H., Weil, C., Springer, N., Burtner, C., Young, K., Bowers, E., Codomo, C.A., Enns, L.C., 
Odden, A.R., Greene, E.A., Comai, L and Henikoff, S. 2004. Discovery of induced point mutations in maize genes by TILLING. BMC Plant Biology 4: 12. Colbert, T., Till, B.J., Tompa, R., Reynolds, S., Steine, M.N., Yeung, A.T., McCallum, C.M., Comai, L. and Henikoff, S. 2001. High throughput screening for induced point mutations. Plant Physiology 126: 480-484.

Comai, L., Young, K., Till, B.J., Reynolds, S.H., Greene, E.A., Codomo, C.A., Enns, L.C and Johnson, J.E., Burtner, C., Odden, A.R. and Henikoff,S.2004. Efficient discovery of DNA polymorphisms in natural populations by Ecotilling. Plant Journal 37: 778786.

Frerichmann, S. L., Kirchhoff, M., Müller, A. E., Scheidig, A. J., Jung, C. and Kopisch-Obuch, F. J. 2013. Eco TILLING in Beta vulgaris reveals polymorphisms in the FLC-like gene $B v F L 1$ that are associated with annuality and winter hardiness. $B M C$ Plant Biol.

Gilchrist, E.J., Haughn, G.W., Ying, C.C., Otto, S.P., Zhuang, J., Cheung, D., Hamberger, B., Aboutorabi, F., Kalynyak, T., Johnson, L, Bohlmann, J., Ellis, B., Douglas, C.J. and Cronk, Q.C. 2006. Use of Ecotilling as an efficient SNP discovery tool to survey genetic variation in wild populations of Populus trichocarpa. Molecular Ecology 15(5):1367-1378.

Gilchrist, E.J., O’Neil, N.J., Rose, A.M., Zetka, M.C., Haughn, G.W.2006. TILLING is an effective reverse genetics technique for Caenorhabditis elegans. BMC Genomics 7: 262.

Henikoff, S., Till, B.J. and Comai, L. 2004. TILLING: Traditional mutagenesis meets functional genomics. Plant Physiology 135: 630-636.
Ibiza, V. P., Cañizares, J. and Nuez, F. 2010. Eco TILLING in Capsicum species: searching for new virus resistances. BMC Genomics 11:631.

Lightner, J. and Caspar T (1998). Seed mutagenesis of Arabidopsis. Arabidopsis Protocols: 91-102.

McCallum C. M., Comai L., Greene E.A and Henikoff, S. 2000. Targeting induced local lesions in genomes TILLING for plant functional genomics. Nat Biotechnology 18:455-457.

McCallum, C. M., Comai, L., Greene, E.A., Henikoff, S. 2000. Targeted screening for induced mutations. Nat. Biotechnology 18: 455-457.

Mejlhede, N., Kyjovska, Z., Backes, G., Burhenne, K., Rasmussen, S. K. and Jahoor, A. 2006. Eco TILLING for the identification of allelic variation in the powdery mildew resistance genes mlo and Mla of barley. Plant Breeding 125, 461-467.

Nagy, A., Perrimon, N., Sandmeyer, S. and Plasterk, R. 2003. Tailoring the genome: The power of genetic approaches. Nat Genet 33: 276-284.

Negrao, S., Courtois, B., Ahmadi, N., Abreu, I., Saibo, N. and Oliveira, M. M. 2011. Recent updates on salinity stress in rice: from physiological to molecular responses. Crit. Rev. Plant Science. 30, 329-377.

Perry, J. A., Wang, T. L., Welham, T. J., Gardner, S., Pike, J. M., Yoshida, S., et al., 2003. A TILLING reverse genetics tool and a web-accessible collection of mutants of the legume Lotus japonicus. Plant Physiol. 131, 866-871.

Raghavan, C., Naredo, M. E. B., Wang, H., Atienza, G., Liu, B., Qiu, F., et al., 2007. Rapid method for detecting SNPs on agarose gels and its application in candidate gene mapping. Mol. Breed. 19, 87-101. 
Rashid, M., He, G., Guanxiao, Y. and Khurram, Z. 2011. Relevance of tilling in plant genomics. Australian Journal of Crop Science 5(4): 411-420.

Suzuki, T., Eiguchi, M., Satoh, H., Kumamaru, T., and Kurata, N. 2005. A modified TILLING system for rice mutant screening. Rice Genet. News Lett. 22, 89-91.

Till, B.J., Colbert, T., Tompa, R., Enns, L.C., Codomo, C.A., Johnson, J.E., Reynolds, S.H., Henikoff, J.G., Greene, E.A., Steine, M.N., Comai, L. and Henikoff, S.2003.High-throughput TILLING for functional genomics. In Grotewold $E$ (ed) Methods in Molecular Biology 236: 205-220.

Till, B.J., Zerr, T., Bowers, E., Greene, E.A., Comai, L. and Henikoff S.2006. High throughput discovery of rare human nucleotide polymorphisms by Ecotilling. Nucleic Acids Research 34: 99.

Till, B. J., Jankowicz-Cieslak, J., Sagi, L., Huynh, O. A., Utsushi, H., Swennen, R. 2010. Discovery of nucleotide polymorphisms in the Musa gene pool by Eco TILLING. Theoretical Applied Genetics 121, 1381-1389.

Till, B. J., Zerr, T., Comai, L., and Henikoff, S. 2006. A protocol for TILLING and Eco TILLING in plants and animals. Nat. Protoc. 1, 2465-2477.
Till, B.J., Cooper, J., Tai, T.H., Colowit, P., Greene, E.A., Henikoff, S. and Comai, L. 2007. Discovery of chemically induced mutations in rice by TILLING. BMC Plant Biology 7: 19.

Till, B.J., Reynolds, S.H., Greene, E.A., Codomo, C.A., Enns, L.C., Johnson, J.E., Burtner, C., Odden, A.R., Young, K., Taylor, N.E., Henikoff, J.G., Comai, L., Henikoff, S. 2003. Large-scale discovery of induced point mutations with high-throughput TILLING. Genome Research 13: 524-530.

Yang, W., Bai, X., Kabelka, E., Eaton, C., Kamoun, S., van der Knaap, E., et al., 2004. Discovery of single nucleotide polymorphisms in Lycopersicon esculentum by computer aided analysis of expressed sequence tags. Mol. Breed. 14, 21-34.

Yu, S., Liao, F., Wang, F., Wen, W., Li, J., Mei, H., et al., 2012. Identification of rice transcription factors associated with drought tolerance using the Eco TILLING method. PLOS ONE 7: e30765.

Zhanguo, X., Wang, M.L., Barkley, N.A., Burow, G., Franks, C., Pederson, G and Burke, J. 2008. Applying genotyping (TILLING) and phenotyping analyses to elucidate gene function in a chemically induced sorghum mutant population. BMC Plant Biology 8:103.

\section{How to cite this article:}

Azra Khan, Ishfaq Abidi, M.A. Bhat, Z.A. Dar, Gowhar Ali, Asif B. Shikari and Khan, M.A. 2018. Tilling and Eco-Tilling - A Reverse Genetic Approach for Crop Improvement. Int.J.Curr.Microbiol.App.Sci. 7(06): 15-21. doi: https://doi.org/10.20546/ijcmas.2018.706.003 